\title{
Cathodic electrodeposition of SnS thin films from aqueous solution
}

\begin{abstract}
SnS thin films were prepared by cathodic electrodeposition on ITO/glass and Ti substrates from a solution containing $\mathrm{SnCl} 2$ and thiosulphate ions. Cyclic voltammetry experiments were performed to elucidate the electrodic processes occurred when potentials were applied and to determine the optimum potential for electrodeposition. The photoactivity of the deposited films and their conduction types were evaluated using the photoelectrochemical technique. The bandgap energy and type of optical transitions were determined from optical absorbance data. Structural and compositional analysis were accomplished using X-ray diffractometry, electron dispersive analysis of X-ray, and X-ray photoelectron spectroscopy. The morphology of the films were examined using scanning electron microscopy.
\end{abstract}

Keyword: Cathodic electrodeposition; Thin films 